\title{
Protocol for a Korean Multicenter Prospective Cohort Study of Active Surveillance or Surgery (KoMPASS) in Papillary Thyroid Microcarcinoma
}

\begin{abstract}
Min Ji Jeon ${ }^{1, *}$, Yea Eun Kang ${ }^{2, *}$, Jae Hoon Moon ${ }^{3}$, Dong Jun Lim ${ }^{4}$, Chang Yoon Lee ${ }^{5}$, Yong Sang Lee Sun Wook Kim ${ }^{7}$, Min-Hee Kim ${ }^{8}$, Bo Hyun Kim ${ }^{9}$, Ho-Cheol Kang ${ }^{10}$, Minho Shong ${ }^{2}$, Sun Wook Cho ${ }^{11}$, Won Bae Kim ${ }^{1}$

${ }^{1}$ Department of Internal Medicine, Asan Medical Center, University of Ulsan College of Medicine, Seoul; ${ }^{2}$ Department of Internal Medicine, Chungnam National University Hospital, Daejeon; ${ }^{3}$ Department of Internal Medicine, Seoul National University Bundang Hospital, Seoul National University College of Medicine, Seongnam; ${ }^{4}$ Department of Internal Medicine, Seoul St. Mary's Hospital, College of Medicine, The Catholic University of Korea, Seoul; ${ }^{5}$ Department of Radiology, National Cancer Center, Goyang; ${ }^{6}$ Department of Surgery, Thyroid Cancer Center, Gangnam Severance Hospital, Institute of Refractory Thyroid Cancer, Yonsei University College of Medicine; ${ }^{7}$ Department of Medicine, Thyroid Center, Samsung Medical Center, Sungkyunkwan University School of Medicine; ${ }^{8}$ Department of Internal Medicine, Eunpyeong St. Mary's Hospital, College of Medicine, The Catholic University of Korea, Seoul; ${ }^{9}$ Department of Internal Medicine, Biomedical Research Institute, Pusan National University Hospital, Busan; ${ }^{10}$ Department of Internal Medicine, Chonnam National University Hwasun Hospital, Hwasun; ${ }^{11}$ Department of Internal Medicine, Seoul National University Hospital, Seoul, Korea
\end{abstract}

Background: A Korean Multicenter Prospective cohort study of Active Surveillance or Surgery (KoMPASS) for papillary thyroid microcarcinomas (PTMCs) has been initiated. The aim is to compare clinical outcomes between active surveillance (AS) and an immediate lobectomy for low-risk PTMCs. We here outline the detailed protocol for this study.

Methods: Adult patients with a cytopathologically confirmed PTMC sized 6.0 to $10.0 \mathrm{~mm}$ by ultrasound (US) will be included. Patients will be excluded if they have a suspicious extra-thyroidal extension or metastasis of a PTMC or multiple thyroid nodules or other thyroid diseases which require a total thyroidectomy. Printed material describing the prognosis of PTMCs, and the pros and cons of each management option, will be provided to eligible patients to select their preferred intervention. For the AS group, thyroid US, thyroid function, and quality of life (QoL) parameters will be monitored every 6 months during the first year, and then annually thereafter. Disease progression will be defined as a $\geq 3 \mathrm{~mm}$ increase in maximal diameter of a PTMC, or the development of new thyroid cancers or metastases. If progression is detected, patients should undergo appropriate surgery. For the lobectomy group, a lobectomy with prophylactic central neck dissection will be done within 6 months. After initial surgery, thyroid US, thyroid function,

Received: 27 October 2020, Revised: 9 December 2020,

Accepted: 12 January 2021

Corresponding authors: Won Bae Kim

Division of Endocrinology and Metabolism, Department of Internal Medicine, Asan Medical Center, University of Ulsan College of Medicine, 88 Olympic-ro 43-gil, Songpa-gu, Seoul 05505, Korea

Tel: +82-2-3010-3913, Fax: +82-2-3010-6962, E-mail: kimwb@amc.seoul.kr

Sun Wook Cho

Department of Internal Medicine, Seoul National University Hospital, 101

Daehak-ro, Jongno-gu, Seoul 03080, Korea

Tel: +82-2-2072-4761, Fax: +82-2-762-2292, E-mail: swchomd@snu.ac.kr

\section{Copyright $\left({ }^{2021}\right.$ Korean Endocrine Society}

This is an Open Access article distributed under the terms of the Creative Commons Attribution Non-Commercial License (https://creativecommons.org/ licenses/by-nc/4.0/) which permits unrestricted non-commercial use, distribution, and reproduction in any medium, provided the original work is properly cited.

*These authors contributed equally to this work. 
serum thyroglobulin (Tg), anti-Tg antibody, and QoL parameters will be monitored every 6 months during the first year and annually thereafter. Disease progression will be defined in these cases as the development of new thyroid cancers or metastases.

Conclusion: KoMPASS findings will help to confirm the role of AS, and develop individualized management strategies, for lowrisk PTMCs.

Keywords: Active surveillance; Watchful waiting; Thyroid cancer, papillary; Cohort studies; Comparative study

\section{INTRODUCTION}

A significant increase in the incidence of papillary thyroid cancer, especially papillary thyroid microcarcinoma (PTMC), has been reported worldwide [1]. Excellent clinical outcomes have been reported for PTMC patients due to the indolent nature [2]. As a result, active surveillance (AS) instead of immediate surgery has been suggested as an alternative management approach for low-risk PTMCs [3]. AS involves a life-long regular followup of the PTMC status with appropriate surgical treatment provided only when disease progression becomes apparent [4-6]. Since the introduction of AS for PTMCs by Japanese surgeons [3], many retrospective observational studies have suggested that this is a safe and effective approach for the management of low-risk PTMCs [7-9]. In a prior meta-analysis of PTMC patients, the pooled proportion of 5-year tumor size enlargements $\geq 3 \mathrm{~mm}$ was found to be only $5.3 \%$, and that of 5-year lymph node (LN) metastasis only $1.6 \%$ during AS [10].

Recent American Thyroid Association guidelines recommend a lobectomy as a standard surgical treatment for low-risk PTMCs unless there are clear indications to remove the contralateral lobe [11]. A lobectomy does not pose a significantly higher risk of recurrence than a total thyroidectomy according to a previous retrospective study of PTMCs [12]. Furthermore, the incidence of postoperative complications following a lobectomy is very low by high-volume surgeons [12,13], and the incidence of postoperative hypothyroidism in patients who have undergone a lobectomy is only approximately $20 \%$ [14]. These findings have suggested that a lobectomy may be safer and more effective than AS in PTMC patients [15]. However, only single-arm retrospective studies of AS have been conducted so far, and no prior reports have compared the clinical outcomes of AS with those of lobectomy in PTMC populations.

To address this issue and compare the clinical outcomes of AS with those of an immediate lobectomy in PTMC, a Korean Multicenter Prospective cohort study of Active Surveillance or Surgery (KoMPASS) in low-risk PTMC cases has been initiated. KoMPASS aims to compare the disease progression rate, quality of life (QoL), and cost-effectiveness between AS and lobectomy to confirm the efficacy of the AS approach in the management of low-risk PTMCs. Factors associated with disease progression during AS, socioeconomic and QoL parameters affecting the informed decision-making in terms of intervention with their changes during follow-up, appropriate imaging follow-up strategies during AS, and differences in surgery-related factors such as the surgical extent, postoperative staging and postoperative complications between immediate and delayed surgery will also be evaluated. We hear introduce the details of the KoMPASS protocol.

\section{METHODS}

\section{Study design}

KoMPASS will be a nationwide prospective observational cohort study of low-risk PTMCs. Eleven referral hospitals in Korea are participating and the study protocol was approved by the ethics committee of each institution: Asan Medical Center (20191541), Seoul National University Bundang Hospital (B-1912/ 583-304), Seoul St. Mary's Hospital (XC20ONDE-0053K), the National Cancer Center (NCC2020-0123), Gangnam Severance Hospital (3-2020-0077), Seoul National University Hospital (1911-149-1083), Samsung Medical Center (2019-11-140), Eunpyeong St. Mary's Hospital (XC20ONDE0053P), Pusan National University Hospital (2004-03-089), Chonnam National University Hwasun Hospital (CNUHH-2020-086), and Chungnam National University Hospital (2019-11-054). The protocol of this study is registered at the Clinical Research Information Service of Korea Centers for Disease Control and Prevention (KCT0004935). Patients will be included from August 2020 to August 2023, and follow-up data will be collected until August 2024.

\section{Study population and enrollment}

Low-risk PTMCs which can be treated by lobectomy are considered eligible. Patients with an eligible PTMC will be offered the printed material containing a detailed description of the clinical outcomes associated with a low-risk PTMC, and the pros 
and cons of AS and lobectomy. After careful consideration, patients will be asked to select their preferred treatment approach. Up to 3 months will be given to patients to make this decision (i.e., an informed decision process). When patients decide upon their treatment option, the physicians will explain the KoMPASS protocol and informed consent to participate will be obtained. The clinical and socioeconomic characteristics of the study patients, their initial PTMC status including ultrasound (US) and computed tomography (CT) results, laboratory findings including thyroid function, and QoL parameters assessed by two questionnaires will be collected at the time of enrollment (Fig. 1). The Korean versions of the questionnaires to be used in KoMPASS have been validated in previous studies [16,17].

Inclusion criteria

- Adult patients (aged 19 to 80) with a thyroid nodule which fulfils all of the following criteria:

- A maximum diameter of between $6.0 \mathrm{~mm}$ and $10.0 \mathrm{~mm}$ measured by US.

- Diagnosed as suspicious for malignancy (Bethesda category $\mathrm{V}$ [18]) or as a malignancy (Bethesda category VI) by either fine needle aspiration or core needle biopsy.

- In cases of Bethesda category V lesions, the nodule should show highly suspicious US features in accordance with the Korean Thyroid Imaging Reporting and Data System (KTIRADS) [19] or should harbor a BRAF V600E mutation.

\begin{tabular}{|c|c|}
\hline \multicolumn{2}{|c|}{$\begin{array}{l}\text { - Need cytologic or histopathologic confirmation for PTMC } \\
\text { - Evaluate thyroid US, CT, thyroid function to confirm PTMC status } \\
\text { and surgical extent }\end{array}$} \\
\hline \multicolumn{2}{|c|}{$\begin{array}{l}\text { Informed decision making: AS vs. immediate surgery (lobectomy) } \\
\text { - Collect socioeconomic characteristics and QoL parameters }\end{array}$} \\
\hline AS group & Lobectomy group \\
\hline \multirow{2}{*}{$\begin{array}{l}\text { Follow-up of PTMC status (thyroid } \\
\text { US), thyroid function, and QoL param- } \\
\text { eters every } 6 \text { months during the first } \\
\text { year and annually thereafter } \\
\text { - If disease progression was detected, } \\
\text { patients should undergo appropriate } \\
\text { surgery. }\end{array}$} & $\begin{array}{l}\text { Lobectomy \& prophylactic CND } \\
\text { within } 6 \text { months from decision }\end{array}$ \\
\hline & $\begin{array}{l}\text { Follow-up of PTMC status (thyroid US), } \\
\text { thyroid function, Tg and TgAb, and QOL } \\
\text { parameters every } 6 \text { months during the } \\
\text { first year and annually thereafter }\end{array}$ \\
\hline
\end{tabular}

Fig. 1. Flow diagram of the study process. PTMC, papillary thyroid microcarcinoma; US, ultrasound; CT, computed tomography; AS, active surveillance; QoL, quality of life; CND, central neck dissection; Tg, thyroglobulin; TgAb, anti-Tg antibody.

\section{Exclusion criteria}

- Patients who can't understand or don't agree to the study protocol.

- Pregnant women.

- Patients with a history of head and neck cancer, actively receiving treatment for other cancers, or with any uncontrolled chronic disease.

- Patients with multiple thyroid nodules or another thyroid disease requiring a total thyroidectomy.

- PTMC with a suspicion of a cervical LN metastasis determined by either US or thyroid protocol CT [6].

- PTMC with a suspicion of distant metastasis, with clinical symptoms/signs of extra-thyroidal extension of the tumor, or showing invasion to recurrent laryngeal nerve [6].

\section{Follow-up protocol}

For the PTMC patients undergoing AS, thyroid US, thyroid function, and QoL parameters will be monitored every 6 months during the first year, and then annually thereafter [3]. If disease progression is detected, patients should undergo the appropriate surgery. For patients deciding to undergo a lobectomy, a lobectomy with prophylactic central neck dissection will be done within 6 months of this decision. After the initial surgery, thyroid US, thyroid function, serum thyroglobulin (Tg), anti-Tg antibody, and QoL parameters will be monitored every 6 months during the first year, and then annually thereafter. During follow-up, thyroid function needs to be maintained in an euthyroid status.

Patients undergoing AS will be allowed to change their treatment option at any time during follow-up, i.e., they can elect to undergo surgery. However, any patient that changes their treatment option within 6 months of the initial enrollment will be excluded from the study.

\section{Study outcomes}

The primary outcome of the KoMPASS study is progressionfree survival. Disease progression in the study patients undergoing AS will be defined by any of the following criteria: (1) a maximum PTMC size increase of $\geq 3 \mathrm{~mm}$ [3]; (2) a cytopathological diagnosis of a new thyroid cancer lesion; (3) a cytopathological diagnosis of a cervical LN metastasis; or (4) clinical or radiological suspicion of a distant metastasis. Disease progression after a lobectomy will be defined by any of the following: (1) a cytopathological diagnosis of a new lesion in the remnant thyroid; (2) a cytopathological diagnosis of a cervical LN metastasis; or (3) a clinical or radiological suspicion of a distant 
metastasis.

For secondary outcomes, we aim to evaluate overall survival, socioeconomic status and the QoL parameters of the enrolled patients. We will also examine the cost-effectiveness of the two different management approaches, the risk factors for disease progression during AS (i.e., the age and sex of the patients, US features of nodules, the tumor volume [TV], and the TV doubling time [20]), any reasons for a delayed surgery, appropriate imaging follow-up strategies during AS, and differences in surgery-related factors such as the surgical extent, postoperative staging and postoperative complications between immediate surgery and delayed surgery.

\section{Sample size calculation}

According to previous retrospective studies and meta-analyses of PTMCs, the disease progression rate associated with AS is about $6.9 \%$ [10] and that for an immediate lobectomy is around $1.6 \%$ [21]. We used inequality hypothesis for two survival curves using a Cox's proportional hazards model. The hazard ratio for the surgery group will be set to 0.54 based on our prior experience. The expected enrollment ratio for AS and surgery group is estimated to be about 1:1. The alpha-error will be set to 0.05 based on a two-sided effect and the power will be set to $80 \%$. With these assumptions, the sample size per group has been estimated at 973 (total 1,946) via the Power Analysis Sample Size function of PASS 15 software (NCSS LCC., Kaysville, UT, USA). We aim therefore to recruit a total of 2,000 PTMC patients into the KoMPASS trial.

\section{Data analysis}

Continuous data will be presented as a mean \pm standard deviation or median with interquartile range. Categorical data will be presented as numbers with percentages. A Cox proportional hazard models will be used to evaluate survival outcomes. Both intent to treat and per protocol analysis will be conducted. To reduce possible biases, propensity score matching or inverse probability of treatment weighting methods will be used. Variables measured multiple times during follow-up will be compared with the paired $t$ test in accordance with the clinical management used. A significant level will be set at 0.05 and $R$ version 3.1.0 and R libraries survival, car, prodlim, and Cairo software will be used to analyze the data (R Foundation for Statistical Computing, http://www.R-project.org).

\section{Dissemination policy}

The scientific integrity of this project requires that data from all centers should be analyzed and reported. Thus, an individual center is not expected to report the data collected from its center alone. Topics suggested for publication will be circulated to the principal investigators of all centers. The lead author of the study should be the person who suggested the topic and co-authors should be decided according to the authorship guidelines.

\section{DISCUSSION}

AS has only been introduced recently for the management of PTMC cases, and most of the evidence to date for the effectiveness of this approach has come from single-arm, short-term, retrospective studies $[3,8,9,22]$. Some prospective comparative studies have been conducted for AS interventions in PTMC patients, but the results have not been reported yet $[6,23]$. The strengths of the KoMPASS protocol are that it follows the recent guidelines and protocols of some of the seminal reports on PTMC $[3,11,19,24]$ and will be a head-to-head comparison of AS and lobectomy, which is the standard surgical intervention used for low-risk PTMCs. We aim over the next 4 years to compare various effects of AS and lobectomy on the clinical outcomes of PTMC patients, including the oncologic and surgical outcomes, QoL, and cost-effectiveness. We will also evaluate the appropriateness of different imaging follow-up strategies during AS.

The KoMPASS protocol is primarily characterized by its inclusion and exclusion criteria, which limit the enrollment eligibility to subjects that can be treated by lobectomy, its use of routine CT examinations to confirm the PTMC status of the patients at the time of enrollment [25], and its requirement that patients receive detailed and adequate information to enable informed decision-making regarding their study participation and choice of treatment. The PTMC patients select their preferred clinical management approach, i.e., AS or immediate lobectomy, by themselves after careful consideration of this relevant information. We will evaluate the socioeconomic characteristics and QoL parameters of our enrolled patients, as this can potentially affect their informed decision-making, and assess any QoL changes during follow-up in accordance with the selected management option. Cost-effectiveness is also an important issue in relation to AS management of PTMC and has been reported to be dependent on the patient-specific QoL in a previous study [26]. The KoMPASS protocol thus includes appropriate questionnaires for analyzing the cost-effectiveness of AS in comparison with a lobectomy.

A potential limitation of this study protocol is the possibility Copyright (C) 2021 Korean Endocrine Society 
of a selection bias. The patients will select the management option for the PTMC, and this process cannot be controlled by the investigators. We will however statistically adjust for possible bias using propensity score matching or weighting methods. Patients who subsequently change to the surgery option, even without evidence of disease progression, during AS could also affect the study findings. A previous meta-analysis of PTMC cases has reported that disease progression was not the reason for delayed surgery in two-thirds of the patients who had initially been undergoing AS [10]. Notably however, a recent report has indicated that the 5-year cumulative surgery conversion rates during AS have been significantly decreased from $12.3 \%$ prior to November 2009 to $4.2 \%$ after December 2009 [27].

Notwithstanding the aforementioned limitations, KoMPASS will be the first nationwide large-scale prospective study of patients with low-risk PTMCs in Korea. Eleven referral hospitals in various regions of Korea will be participating, which will enable a large patient population with various characteristics to be recruited. The aim is to enroll 2,000 PTMC patients within the next 3 years and collect detailed follow-up data for this cohort. Furthermore, a multidisciplinary team of thyroid cancer specialists including endocrinologists, thyroid surgeons and thyroid radiologists will be participating as investigators and can perform optimal management and follow-ups for the PTMC patients in the study. The findings from KoMPASS will help us to develop more optimal management options for PTMC that accord with the clinical, socioeconomic, and radiologic characteristics of the affected patients and will provide definitive evidence that may validate the role of AS in the management of low-risk PTMCs.

In conclusion, we have commenced a nationwide multicenter prospective cohort study for low-risk PTMCs in Korea that compares AS and surgery (lobectomy) and will be known as KoMPASS. The results of this prospective investigation will confirm the role of AS and help to develop individualized management strategies for low-risk PTMC patients.

\section{CONFLICTS OF INTEREST}

No potential conflict of interest relevant to this article was reported.

\section{ACKNOWLEDGMENTS}

This research was supported by a grant of the Korea Health Technology R\&D Project through the Korea Health Industry Development Institute (KHIDI), funded by the Ministry of
Health and Welfare, Republic of Korea (grant numbers: HC19C0481, HC19C0215).

The part of the manuscript was reported as an abstract of the symposium at the 2020 annual congress of Korean Surgical Society.

\section{AUTHOR CONTRIBUTIONS}

Conception or design: M.J.J., J.H.M., D.J.L.,C.Y.L., Y.S.L., S.W.K., M.H.K., B.H.K., H.C.K., M.S. Drafting the work or revising: M.J.J., Y.E.K. Final approval of the manuscript: S.W.C., W.B.K.

\section{ORCID}

Min Ji Jeon https://orcid.org/0000-0002-1297-107X

Yea Eun Kang https://orcid.org/0000-0002-2012-3716

Sun Wook Cho https://orcid.org/0000-0002-7394-3830

Won Bae Kim https://orcid.org/0000-0003-4544-1750

\section{REFERENCES}

1. Davies L, Welch HG. Current thyroid cancer trends in the United States. JAMA Otolaryngol Head Neck Surg 2014; 140:317-22.

2. Jeon MJ, Kim WG, Choi YM, Kwon H, Lee YM, Sung TY, et al. Features predictive of distant metastasis in papillary thyroid microcarcinomas. Thyroid 2016;26:161-8.

3. Ito Y, Miyauchi A, Kihara M, Higashiyama T, Kobayashi K, Miya A. Patient age is significantly related to the progression of papillary microcarcinoma of the thyroid under observation. Thyroid 2014;24:27-34.

4. Jeon MJ, Kim WG, Chung KW, Baek JH, Kim WB, Shong YK. Active surveillance of papillary thyroid microcarcinoma: where do we stand? Eur Thyroid J 2019;8:298-306.

5. Haser GC, Tuttle RM, Urken ML. Challenges of active surveillance protocols for low-risk papillary thyroid microcarcinoma in the United States. Thyroid 2016;26:989-90.

6. Brito JP, Ito Y, Miyauchi A, Tuttle RM. A clinical framework to facilitate risk stratification when considering an active surveillance alternative to immediate biopsy and surgery in papillary microcarcinoma. Thyroid 2016;26:144-9.

7. Kwon H, Oh HS, Kim M, Park S, Jeon MJ, Kim WG, et al. Active surveillance for patients with papillary thyroid microcarcinoma: a single center's experience in Korea. J Clin Endocrinol Metab 2017;102:1917-25.

www.e-enm.org 
8. Oh HS, Ha J, Kim HI, Kim TH, Kim WG, Lim DJ, et al. Active surveillance of low-risk papillary thyroid microcarcinoma: a multi-center cohort study in Korea. Thyroid 2018; 28:1587-94.

9. Tuttle RM, Fagin JA, Minkowitz G, Wong RJ, Roman B, Patel S, et al. Natural history and tumor volume kinetics of papillary thyroid cancers during active surveillance. JAMA Otolaryngol Head Neck Surg 2017;143:1015-20.

10. Cho SJ, Suh CH, Baek JH, Chung SR, Choi YJ, Chung KW, et al. Active surveillance for small papillary thyroid cancer: a systematic review and meta-analysis. Thyroid 2019;29:1399408.

11. Haugen BR, Alexander EK, Bible KC, Doherty GM, Mandel SJ, Nikiforov YE, et al. 2015 American Thyroid Association management guidelines for adult patients with thyroid nodules and differentiated thyroid cancer: the American Thyroid Association guidelines task force on thyroid nodules and differentiated thyroid cancer. Thyroid 2016;26:1-133.

12. Kwon H, Jeon MJ, Kim WG, Park S, Kim M, Song DE, et al. A comparison of lobectomy and total thyroidectomy in patients with papillary thyroid microcarcinoma: a retrospective individual risk factor-matched cohort study. Eur J Endocrinol 2017;176:371-8.

13. Hauch A, Al-Qurayshi Z, Randolph G, Kandil E. Total thyroidectomy is associated with increased risk of complications for low- and high-volume surgeons. Ann Surg Oncol 2014;21:3844-52.

14. Park S, Jeon MJ, Song E, Oh HS, Kim M, Kwon H, et al. Clinical features of early and late postoperative hypothyroidism after lobectomy. J Clin Endocrinol Metab 2017;102: 1317-24.

15. Lin JF, Jonker PK, Cunich M, Sidhu SB, Delbridge LW, Glover AR, et al. Surgery alone for papillary thyroid microcarcinoma is less costly and more effective than long term active surveillance. Surgery 2020;167:110-6.

16. Kong SH, Ryu J, Kim MJ, Cho SW, Song YS, Yi KH, et al. Longitudinal assessment of quality of life according to treatment options in low-risk papillary thyroid microcarcinoma patients: active surveillance or immediate surgery (Interim Analysis of MAeSTro). Thyroid 2019;29:1089-96.

17. Kim SH, Ahn J, Ock M, Shin S, Park J, Luo N, et al. The EQ-5D-5L valuation study in Korea. Qual Life Res 2016;25:
1845-52.

18. Cibas ES, Ali SZ. The 2017 Bethesda system for reporting thyroid cytopathology. Thyroid 2017;27:1341-6.

19. Shin JH, Baek JH, Chung J, Ha EJ, Kim JH, Lee YH, et al. Ultrasonography diagnosis and imaging-based management of thyroid nodules: revised Korean Society of Thyroid Radiology Consensus Statement and Recommendations. Korean J Radiol 2016;17:370-95.

20. Oh HS, Kwon H, Song E, Jeon MJ, Kim TY, Lee JH, et al. Tumor volume doubling time in active surveillance of papillary thyroid carcinoma. Thyroid 2019;29:642-9.

21. Kim SK, Park I, Woo JW, Lee JH, Choe JH, Kim JH, et al. Total thyroidectomy versus lobectomy in conventional papillary thyroid microcarcinoma: analysis of 8,676 patients at a single institution. Surgery 2017;161:485-92.

22. Ahn J, Jeon MJ, Song E, Kim TY, Kim WB, Shong YK, et al. Quality of life in patients with papillary thyroid microcarcinoma according to treatment: total thyroidectomy with or without radioactive iodine ablation. Endocrinol Metab (Seoul) 2020;35:115-21.

23. Moon JH, Kim JH, Lee EK, Lee KE, Kong SH, Kim YK, et al. Study protocol of multicenter prospective cohort study of active surveillance on papillary thyroid microcarcinoma (MAeSTro). Endocrinol Metab (Seoul) 2018;33:278-86.

24. Yi KH. The revised 2016 Korean Thyroid Association guidelines for thyroid nodules and cancers: differences from the 2015 American Thyroid Association guidelines. Endocrinol Metab (Seoul) 2016;31:373-8.

25. Lee DH, Kim YK, Yu HW, Choi JY, Park SY, Moon JH. Computed tomography for detecting cervical lymph node metastasis in patients who have papillary thyroid microcarcinoma with tumor characteristics appropriate for active surveillance. Thyroid 2019;29:1653-9.

26. Venkatesh S, Pasternak JD, Beninato T, Drake FT, Kluijfhout WP, Liu C, et al. Cost-effectiveness of active surveillance versus hemithyroidectomy for micropapillary thyroid cancer. Surgery 2017;161:116-26.

27. Sasaki T, Miyauchi A, Ito Y, Kudo T, Kanemura N, Sano T, et al. Marked decrease over time in conversion surgery after active surveillance of low-risk papillary thyroid microcarcinoma. Thyroid 2021;31:217-23. 